\title{
Au miroir de la pseudo-traduction. Ironisation du traduire et traduction de l'ironie
}

\section{David Martens}

Katholieke Universiteit Leuven

Pseudo-translations of literature often produce rich encounters between translation and irony. By faking the formal features and conventions of translation, these texts are ludic means of calling into question, in a parodic mode, certain characteristics of the very type of literature to which they pretend to belong. In so doing, they expose idealized representations of those genre and the practice of translation. This study, based on pseudotranslations by Mérimée and Queneau, examines not only the translation of word-play involving several languages but also those parts of the text which mock the very possibility of translation. This produces situations in which pseudo-translators and real translators alike find themselves forced to forego translating, in its strictest sense, certain passages in order to preserve the ironic play of the original.

\section{Introduction}

Régulièrement conçue comme foncièrement rétive à la traduction, l'ironie apparaît comme un objet spécialement problématique pour les traducteurs et les traductologues. De nature duplice, elle participe fréquemment d'une logique du sous-entendu et repose volontiers sur des jeux de langage réputés relever de la sphère de l' «intraduisible ». Concentrant certains des enjeux fondamentaux qui confrontent le traduire à ses frontières, l'ironie invite par conséquent à penser ce qui constitue sa spécificité dans le domaine des pratiques et des formes scripturaires. À travers l'ironie et son traitement, la traduction serait en effet conduite à traiter avec une part de ses limites et, dans le même temps, à composer avec son identité en tant que geste d'écriture singulier. Réciproquement, la traduction de l'ironie incite à l'examen de certaines des conceptions qui sous-tendent l'appréhension de la traduction.

Dans la mesure où l'ironie est abondamment traduite, son caractère prétendument intraduisible se conçoit en référence à un ensemble de valeurs qui déterminent une conception idéale du traduire, en fonction de laquelle se constitue le domaine de ce qui serait censé échapper à la traduction. Mais qu'en est-il lorsque le texte en jeu joue ironiquement des attendus en vertu desquels se conçoit le champ de l'intraduisible ? En raison du simulacre en fonction duquel elles se donnent à lire, les pseudo-traductions littéraires apparaissent comme un lieu de rencontre privilégié des poétiques de l'ironie et de la traduction. À travers une mise en ouvre factice des caractères formels de la traduction et de ses protocoles paratextuels conventionnels, ces supercheries posent la question de l'identité de la traduction en ironisant 
à plaisir, et en mettant ainsi en question certaines des propriétés caractéristiques du type de textes auquel ils feignent d'appartenir.

De statut et de forme particuliers, ces mystifications engagent un questionnement sur certaines des conventions qui déterminent, de façon plus ou moins latente, la production des traductions et leurs usages. Ils invitent corollairement à une interrogation de certains des présupposés qui régissent la manière dont sont traditionnellement envisagés les rapports de la traduction et de l'ironie, notamment en fonction du paradigme de l'intraduisible. Fondé sur une approche des pseudo-traductions publiées par Mérimée et, surtout, Queneau, ce questionnement ne se bornera dès lors pas à un examen des dispositifs ironiques (et de leur pseudo-traduction) au sein de textes qui ne sont dotés de la qualité de traductions qu'à titre fictif. Si une large part de l'ironie à l'œuvre dans les pseudo-traductions touche au traduire, ces textes ont eux-mêmes été traduits, en ce comprise l'ironie dont le geste traducteur y fait l'objet.

Confrontés à des textes qui mettent en cause leur travail, les traducteurs de pseudo-traductions sont tenus de composer avec des textes sources contraignants et, dans certains cas, particulièrement minés. Comment, en effet, traduire sans dommage des œuvres qui affectent l'axiologie et l'axiomatique qui sous-tendent l'imaginaire de la traduction? En un tel contexte, l'intérêt se porte non seulement sur la traduction de l'ironie portant sur le principe de la traduction, mais aussi sur la façon dont les pseudo-traducteurs et les véritables traducteurs de ces textes mettent en scène leur geste. En raison de l'ironie qu'elles font porter sur leur opération scripturaire, les pseudo-traductions contraignent en effet de façon particulière, au point d'ébranler parfois le mode d'autorité spécifique qui définit la tâche des traducteurs. Une telle situation les incite, à l'occasion, à user d'ironie à leur tour afin de préserver leurs prérogatives, jusqu'à se faire auteurs à leur tour.

\section{L'ironie parodique d'une stratégie mystificatrice}

Les pseudo-traductions s'inscrivent dans la tradition de la mystification littéraire (voir Jeandillou, 1994/2001). Si, au sein de celle-ci, le recours au dispositif de la fausse traduction n'est pas systématique, sa fréquence n'en est pas moins notable. La modernité récente dénombre, en France, plusieurs publications de ce genre, parmi lesquelles, de Mérimée, le Théâtre de Clara Gazul (1825), présenté comme traduit de l'espagnol et La Guzla (1827), qui rassemble des textes supposés traduits de l'illyrique ; les Chansons de Bilitis (1895) de Pierre Louÿs, données à lire comme les œuvres d'une poétesse de l'antiquité grecque ; J'irai cracher sur vos tombes (1946) et les autres romans publiés par le supposé traducteur Boris Vian sous le pseudonyme de Vernon Sullivan ; ou encore On est toujours trop bon avec les femmes (1947) de Raymond Queneau, qui, avec le Théâtre de Clara 
Gazul, est, de ces textes, celui qui engage la parodie la plus élaborée de la traduction ${ }^{1}$.

La fonction de la traduction dans le dispositif mystificateur a souvent été envisagée comme celle d'un leurre plus ou moins plaisant. Ces textes pourraient dès lors passer pour relativement anodins à une réflexion d'ordre traductologique. Cependant, ces simulacres de traduction permettent des transferts culturels (Toury, 1984), notamment des innovations thématiques et/ou formelles au sein d'un champ littéraire déterminé (Lombez, 2005). En dépit de leur indéniable part de farce, ces textes posent des questions cruciales au traduire et à ce qui fait son identité (Apter, 2006, p. 212). L'ironie parodique des fausses traductions cristallise certains des enjeux fondamentaux des relations entretenues par la traduction et l'ironie, notamment au regard de la supposée intraductibilité de cette dernière. Démontrant en actes le caractère codé - c'est-à-dire imitable et donc falsifiable - des traductions, ces œuvres donnent en effet lieu à une configuration textuelle éminemment propice à une poétique de l'ironie.

Lors de sa publication, une fausse traduction se donne dans un premier temps à lire comme autre que ce qu'elle est véritablement. Ce n'est que dans un second temps, une fois la supercherie dévoilée, que ces œuvres sont lues pour ce qu'elles sont : des originaux à vocation mystificatrice. Touchant au statut des textes, cette distinction temporelle constitue un facteur de division potentielle du public des pseudo-traductions, qui recoupe celle fréquemment impliquée par l'ironie. Ces mystifications divisent en effet leur lectorat en deux types différents : entre, d'une part, les actants de la scénographie ironique (Hamon, 1996, pp. 116-126) qui auront connaissance du statut effectif du texte et le liront pour ce qu'il est, à savoir un canular (ils sont susceptibles d'apparaître comme les complices de l'ironiste) et, d'autre part, ceux qui ne percevront pas la supercherie, et en seront ou en auront été les dupes (cette part du public constitue la cible privilégiée de l'ironie potentielle du mystificateur).

Lorsque l'ironie de ces textes se concrétise, elle porte fréquemment sur les enjeux de la traduction, en jouant des présupposés qui lui sont coutumièrement associés. Ainsi, dans la seconde préface (1842) de $\mathrm{La}$ Guzla, après avoir révélé le pot-aux-roses, Mérimée ironise, non sans une certaine complaisance, sur les traductions de certains textes du recueil. La supercherie a de toute évidence fonctionné de façon plus efficace que pour le Théâtre de Clara Gazul, dont la nature n'avait guère trompé le public lors de sa parution. Présenté comme issu d'une culture balkanique relativement méconnue, cet ensemble, qui répondait à un intérêt d'époque pour les cultures orales, a connu un succès d'estime. Le livre a attiré l'attention d'érudits étrangers, dont certains ont publié des traductions de ces textes, manifestement sans connaître le dessous des cartes. 
Deux mois après la publication de la Guzla, M. Bowring, auteur d'une anthologie slave, m'écrivit pour me demander les vers originaux que j'avais si bien traduits.

Puis M. Gerhart, conseiller et docteur quelque part en Allemagne, m'envoya deux gros volumes de poésies slaves traduites en allemand, avec la Guzla traduite aussi, et en vers, ce qui lui avait été facile, disait-il dans sa préface, car sous ma prose il avait découvert le mètre des vers illyriques. Les Allemands découvrent bien des choses, on le sait, et celui-là me demandait encore des ballades pour faire un troisième volume.

Enfin, M. Pouchkine a traduit en russe quelques-unes de mes historiettes, et cela peut se comparer à Gil Blas traduit en espagnol, et aux Lettres d'une religieuse portugaise, traduites en portugais. (Mérimée, 1842/1994, p. 20)

À travers cette scénographie, le futur auteur de Carmen s'assure de mettre le lecteur dans sa poche, en même temps que ses traducteurs lésés en boîte. Manière d'assurer quelque postérité et, peut-être, une certaine promotion de scandale à la nouvelle édition d'un livre dont il souligne au passage le succès modeste. L'auteur énonce, en le rendant public, la partition effective du lectorat qui a procédé de son stratagème mystificateur. En l'occurrence, l'ironie du malicieux préfacier repose sur «le partage de valeurs spécifiques » (Schoentjes, 2001, p. 194) touchant à la traduction. Jamais formulée explicitement, cette convention latente filigrane pourtant le propos (sans quoi l'ironie mériméenne ne fonctionnerait pas) et consiste à considérer qu'une traduction ne se traduirait pas.

\section{Comment (ne pas) traduire une (fausse) traduction ${ }^{2}$}

Dans «La tâche du traducteur», Walter Benjamin considère que si les textes originaux sont susceptibles de se voir traduites à plusieurs reprises, les traductions se révéleraient en revanche intraduisibles (2000, pp. 206261). Le nombre de traductions de traductions réalisées témoignant du contraire, Jaccques Derrida précise, dans un commentaire de cet axiome benjaminien, qu'une traduction ne peut être traduite « en tant que telle » (1998, p. 225). En d'autres termes, si les traductions paraissent intraduisibles en droit, c'est-à-dire en fonction d'un idéal conditionnant la pratique traductive, rien n'empêche qu'elles soient traduites (et elles l'ont été) dans les faits. Selon une telle perspective, une traduction ne serait traduisible qu'en fonction d'un autre, l'original, soit en tant que medium pour une traduction d'un original dont le traducteur ne maîtriserait pas la langue, soit que, à l'instar de celles de Sophocle par Hölderlin, elle ait acquis un statut de quasi original. 
Qu'en est-il des fausses traductions à cet égard ? Qu'advient-il lorsque ces textes subissent l'épreuve de cette étrangeté que constitue la traduction? Une fois révélée leur qualité d'originaux, ils demeurent en partie des traductions, dans la mesure où ils en conservent la forme. Or, comment traduire un texte qui est à la fois un original et une traduction, un original qui, fictivement et formellement, se présente comme une traduction? La question semble de pure rhétorique, dès lors que le dévoilement de la supercherie rend ces textes à leur statut d'originaux véritables. Pourtant, à l'usage, leur traduction ne laisse pas de poser problème, ainsi qu'en témoigne la seconde préface de La Guzla, dans laquelle Mérimée se moque de plusieurs de ses traducteurs, notamment de Pouchkine, dont il suggère que la version russe de certains des textes de La Guzla serait à considérer comme une traduction dans leur langue d'origine supposée.

L'ironie mériméenne se fonde en effet sur le présupposé en vertu duquel une traduction serait intraduisible en tant que telle. Des traductions de «traductions » comme celles de Gerhart et Pouchkine viseraient, à travers les textes de La Guzla, les supposés originaux en langue illyrique et non leur «traduction » française pour elle-même (à en croire Mérimée, les deux premiers lecteurs évoqués lui auraient d'ailleurs demandé de leur procurer l'original). Or, en raison de la méprise en fonction de laquelle ces deux auteurs ont traduit les vers de La Guzla, leurs traductions se sont trouvées d'emblée faussées. La révélation du statut effectif de la supercherie souligne en effet que, si ces traductions se présentent comme des traductions d'une traduction, elles fournissent aux lecteurs allemands et russes une information erronée sur elles-mêmes, puisqu'elles sont, en réalité, des traductions d'un original en français.

L'ironie de cette préface affecte la traduction de Pouchkine de façon plus prononcée que celle de son homologue allemand. Rapprochées par Mérimée de celles de Gil Blas de Santillane en espagnol et de celle des Lettres d'une religieuse portugaise en portugais, elle fait figure de comble. À suivre cette axiologie implicite, cette traduction en russe consisterait en effet en une (ré)écriture de l' « original » effectuée à l'insu du traducteur. Le travail de Pouchkine aurait consisté à traduire ces textes dans la langue de leur version originale fictive $e^{3}$. Selon un renversement typique de l'ironie, l'original serait ici produit, à travers le détour de la mystification, à partir de sa traduction... Par conséquent, le statut de traduction du texte de l'écrivain russe, qu'il justifie toutefois parfaitement, se voit dénié en ce qu'il résulterait d'une (re)production de l'original (imaginaire) à partir de sa supposée traduction ${ }^{4}$.

Certes, les traducteurs abusés peuvent faire l'objet de moqueries plus ou moins faciles. Il n'en reste pas moins que, si les pseudo-traductions constituent des mises en question ironiques du paradigme scripturaire de la traduction, elles sont dans le même temps susceptibles d'être traduites, notamment dans leur langue d'origine supposée (l'espagnol pour Le 
Théâtre de Clara Gazul de Mérimée, l'anglais pour J'irai cracher sur vos tombes). Or, traduire une fausse traduction ne va pas sans mal, même lorsque ces mystifications sont présentées comme les originaux qu'elles sont effectivement. En effet, ces textes piégés posent également des problèmes aux traducteurs qui n'ignorent pas leur véritable nature. La difficulté de ces traductions tient, notamment, à la part d'ironie qu'elles font peser sur le geste du traducteur, tout spécialement s'il s'agit de cibler la langue de leur original imaginaire.

Comment un texte-cible s'y prend-il pour traduire ce texte-source qui, par sa forme, rend périlleuse sa traduction et, dans certains cas, fait peser sur le travail du traducteur une ironie qui en mine par avance la pertinence voire la légitimité ? Un tel projet revêt une part de stimulant défi pour les traducteurs. Il les convie à se confronter à des textes supposant l'impossibilité de leur démarche ou, du moins, faits pour la rendre quelque peu périlleuse. Avec de tels textes, les traducteurs touchent en effet à la qualité d'intraduisible attachée non seulement à la forme des pseudotraductions - qui sont des traductions sans en être - mais aussi, et dans le même temps, à la part d'ironie qui les sous-tend, tout spécialement lorsque celle-ci porte sur le traduire, sur sa possibilité et sur ses attendus, c'est-àdire lorsqu'elle (se) joue d'une certaine conception courante de la traduction.

\section{La loi économique de la langue}

Pour ce qui touche à la traduction de l'ironie, les fausses traductions soulèvent un problème qui procède du feuilletage et de la combinatoire des versions impliquées et de leur statut (fictif) de traduction. Présenté comme traduction, le texte qui fait l'objet de la mystification suppose un original, fût-il imaginaire. Il contraint dès lors son pseudo-traducteur et, du même coup, son véritable traducteur, à composer avec une ironie en amont, celle du supposé original. En d'autres termes, il s'agit, pour les traducteurs des pseudo-traductions, de traduire des segments ironiques de textes censément (et fictivement) déjà traduits. On est toujours trop bon avec les femmes de Queneau complexifie cet écueil. Une large part de l'ironie de ce roman repose sur une combinatoire de langues qui rend d'autant plus délicate la traduction du texte que celui-ci fait à de multiples reprises sa cible des codes de la traduction, qu'il parodie allègrement.

Typiques de la manière de Queneau, dont le modèle joycien est patent (le cri de ralliement des insurgés de ce récit ayant pour cadre l'insurrection irlandaise de 1917 n'est autre que «Finnegans wake!»), certains passages du livre n'ont pas manqué de poser problème(s) à sa traductrice en anglais. Michel Presles, le supposé traducteur de ce roman attribué à une jeune irlandaise nommée Sally Mara, dont il se présente comme le professeur de français, s'est, il est vrai, montré particulièrement 
leste dans la traduction qu'il aurait réalisée du manuscrit de son ancienne élève... Le récit livre en effet à son traducteur en anglais un texte piégé, truffé de jeux de mots conjuguant les langues de Voltaire et de Shakespeare. Or, cette combinatoire, qui consiste à articuler les systèmes de deux langues, interfère avec la possibilité d'une traduction qui ne se heurte pas aux limites traditionnellement assignées à sa démarche.

À en croire Derrida, «[q]uand un "original" parle de sa langue en parlant sa langue il prépare une sorte de suicide à la traduction, comme on dit suicide au gaz ou suicide par le feu » (1990, p. 309) ou, pour le dire autrement, une mise à mort de soi dont la traduction est, en l'occurrence, à la fois l'objet et le moyen. S'il n'est pas possible de traduire en français un segment de texte déjà en français dans un original en langue étrangère, comment, en effet, traduire sans perdre l'effet d'auto-commentaire de l'original ? De façon analogue, citant son « original », une fausse traduction contraint son traducteur dans cette langue à un suicide qui porte sur et en passe par le traduire. Comment traduire un tel texte en rendant compte de la différence des langues qui le caractérise sans se voir ponctuellement contraint à suspendre la traduction au profit de la citation ? Sans compter qu'une telle traduction serait tenue, afin de rendre compte de la différence des idiomes qui caractérise l'original, de notifier ce suspend localisé.

En pareil contexte, la traduction se trouve confrontée à un doublebind en vertu duquel

[i]l faut ou bien se résigner à [...] perdre l'effet, l'économie, la stratégie [de la traduction] [...] ou bien au moins [...] ajouter une glose, du type N.d.T., qui toujours, même dans le meilleur des cas [...], avoue l'impuissance ou l'échec de la traduction [...] rompt avec [...] la loi économique du mot, qui définit l'essence de la traduction au sens strict, de la traduction normale, normée [...]. Partout où l'unité du mot est menacée ou mise en question, ce n'est pas seulement l'opération de la traduction qui se trouve compromise, c'est le concept, la définition et l'axiomatique même, l'idée de la traduction qu'il faut reconsidérer. (Derrida, 2004, p. 565)

Ainsi, lorsque Queneau joue de l'anglais et du français au sein d'une soidisant version française d'un texte écrit à l'origine en «irlandais », la marque de l'anglais laissée par le pseudo-traducteur (Michel Presle) interfère avec la possibilité de réaliser, de l'ensemble du texte, une «traduction au sens strict» de certains segments du roman, à ceci près que le problème intègre la fiction de traduction. En la circonstance, Barbara Whright (1915-2009), traductrice du roman en anglais, se trouve à de multiples reprises confrontée à un problème sans solution à même de répondre de façon parfaitement satisfaisante aux critères de l'idéal de la traduction dont Derrida a mis en évidence la logique aporétique. Certaines inflexions ironiques de la langue d'On est toujours trop bon avec les 
femmes paraissent parfois impossibles à rendre, en particulier lorsque le lexique anglais se trouve pris en charge par les lois de la langue française :

(1) - Un Britiche aux lavatories, dit Mac Cormack. (Queneau, 1947, p. 225, je souligne)

- A Britisher in the lavatory, said Mac Cormack. (Queneau, 1947/2003, p. 44)

La traductrice se trouve contrainte d'adapter en anglais un texte français qui intègre des termes anglais par transposition phonétique dans une graphie francophone. Lorsque Queneau transpose phonétiquement un lexique anglais et en use, qui plus est, en lui appliquant les règles de la grammaire française. En l'occurrence, «lavatory» est employé au pluriel, comme « toilettes » le serait, alors que la version anglaise conserve le singulier. La traduction d'un tel terme en anglais se révèle des plus épineuses au regard d'un idéal de la traduction fondé sur l'économie du mot à mot. La langue anglaise étant précisément celle vers laquelle Whright est supposée traduire, l'opération en est rendue pour le moins hasardeuse, car elle contraint nécessairement à la perte d'un effet de double langue impliqué par l'original, ainsi qu'à celle de la situation singulière du français au regard de la langue anglaise.

(2) [T]out de même, devant ces Républicains, ça le dégoûtait de devoir lutter pour le Roi avec si peu de décor-homme. (Queneau, 1947, p. 194, je souligne)

[E]ven so it disgusted this Frenchman to have to fight these Republicans on behalf of his adopted King with such scant décorhomme. (Queneau, 1947/2003, p. 6, je souligne)

Le choix de la traductrice a consisté à préserver le jeu de mots et son ironie verbale dans sa langue originale et, accessoirement, à ajouter pour désigner un personnage, un qualificatif national de son crû, absent de l'original ${ }^{5}$. Bien que cette option ne relève pas à proprement parler de la traduction elle suspend localement l'opération traductive telle qu'elle se trouve le plus souvent comprise, soit comme la transposition d'un texte d'une langue en une autre -, force est de reconnaître l'art de Whright à maintenir sa version sur la corde raide d'une ironie particulièrement ardue à traduire. À l'instar de l'original pseudo-traducteur, dont elle mime le mode de fonctionnement en d'autres endroits, la traduction préserve. Whright ne dit pas ici la même chose que Queneau dans une autre langue, mais elle fait faire à son texte un équivalent ce que fait l'original, dans la mesure de ce que lui permet la position et le statut de son texte (une traduction) par rapport à l'original.

Dans le même temps que l'échec de l'idéal de la traduction, dans la traduction de Whright, une part de l'ironie du texte fondée sur la 
combinaison de deux langues est parfois perdue, mais pas systématiquement (par exemple lorsqu'elle préserve sans le traduire un jeu de mots de l'original). Certes, la traductrice aurait pu adjoindre des notes destinées à rendre compte de l'ironie de l'original. Mais ces appendices auraient plus que probablement fait tomber l'ironie à plat. La traductrice s'est donc accommodée de ces difficultés et a opté pour un principe d'économie. Dans d'autres cas, lorsque le paratexte pseudo-traductif de la mystification rompt avec l'idéal économique de la traduction, Whright a adopté des stratégies à l'interventionnisme plus manifeste, notamment pour (se) préserver (de) l'ironie générée par la parodie du traduire lorsqu'il touche à ses limites.

\section{Scénographies du suspend traductif}

L'ironie qui sous-tend les fausses traductions atteint fréquemment le principe et la forme de la traduction. Ces supercheries postulent non seulement un original, mais vont en certaines occasions jusqu'à le citer, notamment à travers des notes lexicographiques qui visent à expliciter le sens de certains termes. Ainsi, dans des registres différents, du Théâtre de Clara Gazul, de La Guzla ou d'On est toujours trop bon avec les femmes. Dans la mesure où le dispositif de la traduction participe de la stratégie mystificatrice et se voit doté de la qualité d'original à la faveur du dévoilement de la véritable nature du texte, il s'agit pour un traducteur de pseudo-traduction de traduire l'ensemble du texte, notes comprises. Or, une telle opération ne va pas de soi, car une telle traduction intégrerait en son sein des segments de textes manifestant l'échec du geste scripturaire dont elle procède.

Souhaitant traduire les fausses traductions, tout spécialement dans la langue de leur original imaginaire, en préservant cette «loi économique du mot, qui définit » selon Derrida « l'essence de la traduction au sens strict » (Derrida, 2004, p. 565), un traducteur est conduit à l'annotation de telles notes $^{6}$, afin de maintenir dans la traduction la différence de langues inscrite dans l'original. Ce traducteur devrait en effet préserver le dispositif paratextuel propre à la fausse traduction en annotant les notes qui accompagnent l'original et le citent dans sa langue pour crédibiliser la supercherie. Si cette option retenait ses faveurs, sa traduction frayerait toutefois avec un échec programmé par la structure retorse, voire perverse, du texte à traduire. Une telle stratégie transgresserait la loi économique de la traduction en redoublant un jeu de notes marquant déjà — bien que sur le mode du simulacre - l'échec (en tous les cas le caractère factice) de l'opération traductive en tant que telle.

De façon générale, le redoublement des notes de traducteur est proscrit dans la plupart des traductions de traduction. En ce que c'est l'original qui est visé, ces appendices du texte médian sont le plus souvent 
éliminés. Une traduction en castillan des notes lexicographiques du Théâtre de Clara Gazul supposerait ainsi des notations du type « en castellano en el texto », appelées par les notes de l'édition originale du texte citant des termes de l'original supposé. Placé devant cette alternative, Luis Cernuda, traducteur des pièces en espagnol, a adopté une solution qui, pour être économique (il exclut toute note de ce type), escamote, dans certains cas, la lettre et la forme de l'original. Ainsi, une note relative à un embarras du pseudo-traducteur («Et toi, quand je serai morte...»; note : «Tu te holgarás con ella en la cama comprada de mi dinero. Je ne sais comment traduire » (Mérimée, 1825/1978, p. 209)) disparaît-t-elle tout bonnement dans la traduction (Mérimée, 1825/1933, p. 133).

La savoureuse succession de trois «N. d. T. » qui clôt sur une incongruité en forme de clin d'œil le chapitre XVIII d'On est toujours trop bon avec les femmes est en revanche déterminée par l'idéal de fidélité à la forme plurilinguistique du supposé original. Elle s'articule selon une scénographie qui pousse cette logique jusqu'à l'absurde:

(3) L'esprit irlandais, on le sait, n'obéit pas aux règles du raisonnement cartésien, non plus qu'à celles de la méthode expérimentale. Ni français, ni anglais, mais assez voisin du breton, il procède par « intuition ». Caffrey ne pouvant ouvrir la lourde, eut donc l'ankou ${ }^{1}$ que quelqu'un se trouvait là [...]. Cette anschaunng ${ }^{2}$ lui boucla immédiatement les tripes. Essuyant la sueur qui lui dégoulinait encore de la tronche [...] et, découvrant son devoir d'un seul coup d'un seul ${ }^{3}$, il résolut de rendre compte [...] de la découverte qu'il venait de faire.

Suivent les trois notes de bas de page suivantes :

1. Celticisme pour « intuition». (N. d. T.)

2. Germanisme pour «ankou». (N. d. T.)

3. Gallicisme pour «anschauung ». (N. d. T.) (Queneau, 1947/2003, p. 222)

Jouant du caractère ironique de la répétition (Schoentjes, 2001, p. 169), la structure particulière du texte suggère que l'original fictif traduit par Michel Presle présente trois termes laissés dans leur langue originale, qui témoignent d'une connaissance des langues étrangères laissant à désirer tout en prêtant à sourire. Mais, sous les dehors d'une plaisante pitrerie, l'ironie de ces notes successives s'avère plus incisive qu'il n'y paraît. Son caractère parodique porte son mordant sur les limites de ce qui, dans une conception courante du traduire, définit la traduction en propre, soit le principe d'une unicité linguistique, c'est-à-dire la traduction d'une langue vers une autre langue, sous-tendue par un principe d'économie fondé sur la logique idéale du mot pour mot, refusant les adjonctions autant que les 
restrictions dans le rendu de l'original. Devant cette page rendant problématique son geste, Whright adopte une stratégie de surenchère :

The irish mind, as we know, does not obey the rules of Cartesian logic, any more than it obeys those of experimental methods. Neither French nor English, but fairly close to Breton, it proceeds by "intuition".

As Caffrey couldn't open the door, he therefore had the ankou ${ }^{l}$ that someone was locked in behind it. This anschaunng ${ }^{2}$ immediately tied his guts up in a knot. Wiping awaysweat that wastricklong down his puss, he forgot his egocentric troubles and, discovering his duty d'un seul coup d'un seul', he decided ton inform MacCormack of the discovery he had just made.

Viennent ensuite les trois notes successives:

1. Celticism for "intuition". M.P.

2. Germanism for ankou. M.P.

3. Gallicism for aschauung. M.P. (Also Gallicism for the AngloSaxon cliché "in the twinkling of an eye". B.W.) (Queneau, 1947/2003, pp. 39-40)

Dans l'aparté d'une parenthèse, la traductrice adjoint aux trois notes de l'original une strate supplémentaire, rompant ainsi avec le principe économique régissant la traduction. Ajoutant une référence à l'anglais, langue de sa traduction, à cette série saugrenue de notes qui se traduisent successivement, Whright - membre du Collège de Pataphysique adresse un clin d'œil à son lecteur en évoquant, précisément, "the twinkling of an eye » qui marque la dimension de complicité et de division potentielle du personnel de la scénographie ironique. Elle choisit en outre, pour ce commentaire de son crû, un «cliché » qui, littéralement traduisible en français (« en un clin d'œil»), n'est nullement propre à l'anglais. En outre, le terme choisi (cliché) pour caractériser cette locution censée traduire une locution figée française est de surcroît emprunté au français (Oxford English Dictionnary), tout se passant comme s'il s'agissait d'invalider le geste traducteur au moment même de l'effectuer.

Un jeu de pouvoir, un conflit d'autorité et une interférence de statut entre les signatures traductrice et pseudo-traductrice se noue dans la traduction d'On est toujours trop bon avec les femmes. Whright adjoint ses propres initiales à celles de Michel Presles, qui ne figurent pas dans l'original. Dans la traduction anglaise, les notes de ce dernier sont suivies par ses initiales, alors que dans l'original pseudo-traducteur français, elles sont accompagnées, entre parenthèses, de l'abréviation conventionnelle «N. d. T. ». Dans la version anglaise du texte, cette abréviation, demeurée telle quelle, ou plutôt traduite, renverrait à la traductrice en anglais. Il était 
par conséquent indispensable d'opérer la distinction, ainsi que le fait une seconde fois Whright à l'occasion d'une nouvelle note due à Michel Presle, qu'elle traduit à nouveau en outrepassant ses droits de « stricte » traductrice afin de prolonger (et d'ainsi reprendre à son compte) l'ironie de l'original :

(4) [I]l avait fallu que cette gourde [...] aille s'enfermer dans les vécés au moment tragique et capital, et leur reste sur les bras [...], comme une charge morale, insupportable et peut-être spéculatrice. (Queneau, 1947, p. 259)

Suit la note, appelée par le dernier terme de la citation :

Latinisme (de speculatrix, espionne). Intraduisible en français, langue un peu pauvre, comme chacun sait. (N. d. T.) (Queneau, $1947 / 2003$, p. 259 , note 1 )

Barbara Whright traduit :

[T]his clot [...] had gone and shut herself into de double-you-see at the vital, tragic moment, and here they stuck with her on their hands [...], an unbearable burden, and perhaps even a speculatrix. (Queneau 1947/2003, p. 81)

Et, en note de bas de page :

Latinism (from speculatrix, female spy). Untranslatable into French, wich, as everyone knows, is a somewhat inadequate language. M.P. (English must therefore be a somewhat less inadequate language, for this word can in fact be found in our dictionaries, even if only as an archaism. B.W.) (Queneau 1947/2003, p 81, note 2)

Dans un cas comme dans l'autre, devant ces notes empreintes d'ironie, Whright participe du jeu du traducteur fictif (Presle), soit, en dernière, instance, de celui mis en place par l'auteur (Queneau). Ce faisant, elle se fait elle-même momentanément auteure, à la faveur de l'interruption localisée du geste traducteur à laquelle la contraint la stratégie qu'elle a adoptée pour traduire l'ironie de ces notes. En outre, rajoutant à nouveau une parenthèse signée de ses initiales, et exhibant ainsi un suspens de traduction qui surpasse celui de l' « original », Whright implique à nouveau la langue de sa traduction. Par ce biais, elle ne se borne pas à reproduire le caractère auto-ironique de la note traductive de l'original, mais transpose cette scénographie pour l'adapter à la situation particulière de sa traduction. Pour le dire autrement, elle adapte par surenchère le dispositif ironique de Queneau en lui fournissant un équivalent pour le lecteur anglophone. 
L'ironie constituant l'une des stratégies de défense les plus éprouvées devant l'ironie, confronté à un tel dispositif ironique, le traducteur peut, à l'instar de Barbara Whright, et comme on le dit familièrement, en rajouter une couche, faisant ainsi lui-même acte d'ironie. Il répond ainsi à une double exigence, d'un seul et même geste : rendant compte de l'ironie que le texte original fait porter sur la forme scripturaire de la traduction lorsqu'elle butte sur ses propres limites, il la re-marque et contribue ainsi à la faire remarquer à son lecteur. Ce faisant, il use d'un marqueur d'ironie spécifique, qui consiste à surenchérir sur les notes de Michel Presle, dont les premières sont constitutivement caractérisées et déterminées par la répétition ${ }^{7}$. En l'occurrence, « [1]a traduction de l'ironie exige une certaine manipulation du texte source si l'on veut que la réception de l'ironie dans le texte cible puisse se réaliser » (Lievois, 2006, p. 91).

Confronté à une ironie qui, fondée dans les notes de Queneau sur une répétition parodique, touche à son propre travail, au genre d'écriture qui lui est assigné, que peut le traducteur pour préserver sa fonction et son auctorialité particulières, tout en assumant les impératifs de sa tâche ? L'ironie nécessitant un décodage, ce lecteur qu'est tout traducteur doit témoigner de ce qu'il a bien saisi celle à l'œuvre dans le texte qu'il traduit, sans quoi il encourt le risque de devenir la cible d'une ironie ultérieure, comme celle dont Mérimée fait preuve dans sa préface de 1842 à La Guzla. Il s'agit de témoigner de sa maîtrise de l'ironie, donc de ses compétences de lecteur et de traducteur, en même temps que de sa complicité avec l'auteur de la version originale. Tout se passe comme si l'ironie se présentait devant la traduction et s'offrait au traduire à la fois comme un écueil des plus problématiques, mais aussi comme un vecteur de pouvoir, une valeur cardinale qu'il s'agit de partager sous peine d'en faire les frais.

\section{Conclusion}

Que fait l'ironie à la traduction et réciproquement, que fait la traduction $\grave{a}$ et, dans le même temps, de l'ironie des textes qu'elle traite ? Comme l'a souligné Antonio Lavieri (2006), les mises en scènes fictionnelles du traduire invitent à penser l'épistémologie de la traduction à travers une praxis effective. Les pseudo-traductions nourrissent à cet égard un intéressant feu croisé entre traduction et ironie, de même qu'entre les statuts et prérogatives respectifs de l'auteur et du traducteur qui, dans ces supercheries, tendent à se confondre ponctuellement. Par delà l'indéniable part de pitrerie de supercheries comme celles de Mérimée et de Queneau, ces textes usent de la traduction pour déployer une poétique de l'ironie fondée sur un dispositif mystificateur. Du même coup, ils ironisent sur le traduire, ses formes, ses usages et ses présupposés, et jouent ainsi des représentations associées à ce geste scripturaire à la fois si banal et si énigmatique. 
L'échec ou la contrainte «suicidaire » qui caractérise et borne un certain idéal traditionnel de la traduction témoigne de ce que, bien que les mystifications pseudo-traductives soient, en toute rigueur, des originaux, leur imitation parodique des protocoles de la traduction perturbe, pour les traducteurs de ces textes, le respect du principe économique régissant cette dernière. Devant des pseudo-traductions, toute tentative d'un traducteur pour se plier à la contrainte économique de l'idéal de la traduction faussera son travail en le contraignant à y inscrire au sein l'échec ponctuellement programmé par le texte source. À ce titre, il convient de distinguer entre l'opération traductive au sens strict, notamment lorsqu'elle porte sur des jeux de mots, et ce qui relève de la scénographie de la traduction, de sa mise en scène en tant que geste d'écriture contraint, en particulier lorsqu'il s'agit de composer avec un suspend localisé du traduire.

Marquées par le caractère de traductions des textes qu'elles traduisent, les traductions de fausses traductions sont condamnées à être, elles aussi, de fausses traductions, en dépit de l'habileté parfois remarquable dont leurs traducteurs peuvent faire preuve. Ces supercheries dévoilent l'auctorialité spécifique et problématique qui sous-tend le traduire. En temps normal, selon les critères d'une traduction «idéale », le traducteur est tenu de s'effacer devant le texte à traduire. Devant ces textes qui tendent à pervertir la distinction entre traduction et original et à la faveur desquels un auteur se fait passer (notamment) pour un traducteur, un véritable traducteur paraît tenu, pour assurer son auctorialité particulière, de l'exhiber davantage (sauf à amputer la traduction d'une part de l'original, comme le fait Luis Cernuda dans un geste qui n'est pas moins autoritaire). Ce faisant, il échappe, momentanément du moins, à son geste et à son identité spécifiques, en se faisant auteur à la place de l'auteur.

Les traducteurs qui se sont affrontés à la traduction de pseudotraductions ont en outre dû composer avec l'ironie de ces textes en même temps qu'avec les limites de leur pratique, si bien que l'on peut se demander, au regard de l'idéal de la traduction, si ces traductions de fausses traductions demeurent de bout en bout des traductions? Ces mystifications contraignent à rompre avec l'unicité et l'homogénéité supposées du statut du traducteur et, dans le même temps, de la traduction. Si tout traducteur est à certains égards un auteur - celui de sa traduction - , il doit parfois le devenir de façon plus manifeste lorsque le texte source mime de façon ironique le suspend du traduire stricto sensu. Corollairement, une traduction n'est pas toujours de part en part traduction. Elle s'accompagne fréquemment de dispositifs et de stratégies visant à respecter l'idéal de la traduction, en particulier lorsque celui-ci fait l'objet d'une large part de l'ironie de l'original.

L'ironie des pseudo-traductions, et la façon dont elles contraignent leurs traductions, témoignent du caractère codé de la pratique scripturaire qu'elles parodient. Les problèmes spécifiques posés aux traductions de ce type de supercherie permettent de toucher du doigt le fait que, si l'opération 
traductive est relativement restreinte, les traductions combinent souvent plusieurs types de gestes. Le système de valeurs et de prescriptions qui sous-tend la conception traditionnelle de la traduction ne fonctionne qu'en neutralisant certaines des apories que dévoile l'ironie à l'œuvre au sein des pseudo-traductions. En la matière, tout est question de critères, qui définissent une conception plus ou moins souple ou rigide du traduire et de l'ensemble de ses opérations autorisées. Suicidaires, les pseudo-traductions ne le sont d'ailleurs que localement. Autant dire que leur suicide programmé ne les condamne qu'à de petites morts ponctuelles qui, faisant sortir le traduire de ses gonds, ne vont pas sans une certaine jubilation.

\section{Références}

Apter, E. (2006). Translation with no originals : scandals of textual reproduction. In The Translation zone. A new comparative literature (pp. 210-225). Princeton \& Oxford : Princeton University Press.

Benjamin, W. (2000). La tâche du traducteur. In Euvres (pp. 244-262). T. 1, M., de Gandillac, R., Rochlitz \& P., Rusch (Traduit de l'allemand). Paris: Gallimard. (Première édition publiée en 1923).

Derrida, J. (1998). Des tours de Babel. In Psyché. Inventions de l'autre (pp. 203-235). T. 1. Paris : Galilée (Première édition publiée en 1985).

Derrida, J. (1990). S'il y a lieu de traduire. I. La philosophie dans sa langue nationale (vers une « licterature en françois »). In Du droit à la philosophie (pp. 283-309). Paris : Galilée.

Derrida, J. (2004). Qu'est-ce qu'une traduction «relevante»?. In Mallet, M.-L. \& Michaud, G. (Ed.), Jacques Derrida (pp. 561-576). Paris : L'Herne.

Hamon, P. (1996). L'Ironie littéraire. Essai sur les formes de l'écriture oblique. Paris : Hachette.

Jeandillou, J.-F. (1994). Esthétique de la mystification. Tactique et stratégie littéraire. Paris : Minuit.

Jeandillou, J.-F. (2001). Supercheries littéraires. La Vie et l'œuvre des auteurs supposés. Genève : Droz. (Première édition publiée en 1989).

Lavieri, A. (2006). Mises en scène du traduire: quand la fiction pense la traduction. Transalpina. Études italiennes, 9, 87-101.

Lievois, K. (2006). Traduire l'ironie, entre réception et production. In Trabelsi, M. (Ed.), L'ironie aujourd'hui, lectures d'un discours oblique (pp. 84-94). ClermontFerrand : Presses Universitaires Blaise Pascal.

Lombez, C. (2005). La «traduction supposée » ou : de la place des pseudotraductions poétiques en France. Linguistica Antverpiensa, new series, 4, 107-121.

Martens, D. (à paraître). De la mystification à la fiction. La poétique suicidaire de la fausse traduction. In Klimis, S., Ost, I. \& Vanasten, S. (éd.), Translatio in Fabula. Bruxelles: Presses des Facultés Universitaires Saint-Louis. 
Mérimée, P. (1978). Théâtre de Clara Gazul, In Mallion, J. \& Salomon, P. (Ed.) Théâtre de Clara Gazul, Romans et nouvelles (pp. 1-247). Paris : Gallimard (Bibliothèque de la Pléiade). (Première édition publiée en 1825).

Mérimée, P. (1933). Teatro de Clara Gazul. Comediante española. L. Cernuda. (Traduit en espagnol). Madrid : Escasa-Calpe. (Euvre originale de 1825).

Mérimée, P. (1994). La Guzla ou Choix de poésies illyriques recueillies dans la Dalmatie, la Bosnie et l'Herzégowine, introduction par Fonyi, A. Paris: Kimé. (Euvre originale de 1827; réédition avec préface en 1842).

Queneau, R. (2003). On est toujours trop bon avec les femmes. In Les Euvres complètes de Sally Mara (pp. 188-337). Paris : Gallimard. (Euvre originale de 1947 ; reprise en volume en 1962, sous le nom de Queneau).

Queneau, R. (2003). We always treat women to well. B. Wright (Traduit en anglais). New-York : New-York Review of books. (Euvre originale de 1947)

Schoentjes, P. (2001). Poétique de l'ironie. Paris : Seuil.

Toury, G. (1984). Translation, Literary Translation and Pseudotranslation. In Comparative Criticism, 6, 73-85.

Toury, G. (2005). Enhancing Cultural Changes by Means of fictitious Translations. In Hung, E. (éd.), Translation and Culturel Change (pp. 3-18). Amsterdam \& Philadelphia, Benjamins, 2005.

1 Une étude de la traduction de J'irai cracher sur vos tombes effectuée par Boris Vian en collaboration avec Milton Rosenthal ne manquerait pas d'intérêt dans le cadre d'une étude des relations entre l'ironie et la traduction. Ce texte, son statut et son histoire, posent cependant des questions trop spécifiques que pour pouvoir être abordées dans le cadre de la présente étude.

2 Nous reprenons dans cette sous-section, en les remaniant quelque peu, certains développements d'un article à paraître, qui envisage de façon spéculative des éléments que le présent texte analyse dans certaines de leurs réalisations concrètes (voir bibliographie).

3 L'identification par Mérimée de l'illyrique de La Guzla avec la langue russe paraît toutefois sujette à discussion. L'occasion de faire de l'esprit était sans doute trop tentante...

4 L'axiomatique qui sous-tend le propos de Mérimée pourrait apparaître comme une forme de traduction par anticipation. Fictive quant à son statut, la «traduction » est en effet antérieure à la production de son «original». Sur le plan de la fiction mystifiante du moins, car il est évident que la scénographie ironique mise au point par Mérimée - elle ne fonctionnerait pas sans cela - fait fi de la distinction entre le caractère imaginaire du simulacre de traduction et le statut de traduction véritable de la version russe du texte.

5 Peut-être convient-il d'y voir, en même temps qu'un signe de complicité à l'intention de ses lecteurs, un pied de nez à l'auteur, autrement dit une manière de lui rendre la monnaie de sa pièce. 
6 Ou, à tout le moins, l'adjonction d'une note ou d'une remarque indiquant que l'ensemble des notes figure dans l'original.

7 Sans ces adjonctions ironiques, la traductrice aurait pu passer pou naïve aux yeux du lecteur, pour autant du moins que celui-ci ait perçu l'ironie de l'original. L'une des fonctions de la parenthèse ajoutée par Whright consiste, s'il en était besoin, à assurer davantage encore cette reconnaissance. 